\title{
A SLIP-LINE FIELD SOLUTION FOR PLANE-STRAIN INDENTATION BY AN OBTUSE-ANGLE WEDGE
}

\author{
YILONG BaI \\ Institute of Mechanics, Chinese Academy of Sciences, Peking, China. \\ and \\ BRADLEY DODD
}

Department of Engineering, University of Reading, Whiteknights, Reading RG6 2AY, England

(Received 7 January 1981; in revised form 10 August 1981)

\begin{abstract}
Summary-A slip-line field solution with lines of stress discontinuity is proposed which is shown to be admissible for obtuse-angled wedges with interfacial friction. The dead metal cap solution which is used conventionally when $\theta-\lambda \geqslant \pi / 4$ has a lower load than the corresponding non-dead metal cap solution. This result is referred to as Haddow's paradox. Using the new solution it is shown that, at the point of transition, corresponding to $\theta-\lambda=\pi / 4$, the load coincides with that of the non-dead metal case. Thus Haddow's paradox is inoperative.
\end{abstract}

NOTATION

$\theta$ wedge semi-angle

$\lambda, \psi, \epsilon$ angles in slip-line fields (Fig. 1 and 2)

$\delta_{w}$ tip semi-angle in Fig. 1(a)

$\delta$ tip semi-angle in Fig. 2

$\sigma$ normal stress

$\tau$ shear stress

$k$ yield stress in shear

$c$ depth of indentation

$h$ wedge contact length

$L$ load per unit length of wedge

$\sigma_{i j}$ components of the stress tensor

$n_{j}$ normal to discontinuity surface

$\xi$ angle

\section{INTRODUCTION}

Two slip-line field solutions have been proposed for the plane-strain indentation of a semi-infinite block of rigid perfectly plastic material by partially and completely rough wedges. Fig. 1(a) shows the solution which is admissible for smaller wedge angles. This solution, due to Grunzweig and co-workers [1], is a generalization of the frictionless case $(\lambda=\pi / 4)$ proposed by Hill, Lee and Tupper [2].

Hill[3] suggested that for obtuse-angled wedges $(\theta>\pi / 4)$ with sufficiently high interfacial friction, a dead metal cap solution is applicable, this solution is shown in Fig. 1(b). Johnson et al. [4] showed that the dead metal cap solution was possible when the tip of the wedge in the non-dead metal cap solution becomes overstressed. These researchers also showed that the loads required for the solution in Fig. 1(b) were slightly less than those obtained for the non-dead metal cap solution with sticking friction $(\lambda=0)$. Also it was observed that the critical coefficients of friction for the dead metal case were slightly less than the maximum admissible values for the non-dead metal case.

Haddow [5] completed more extensive calculations for these two solutions and confirmed the findings of Johnson $e t$ al. Haddow referred to these results as a wedge indentation paradox. The discontinuous jump in load between the two solutions at the transition point is now called Haddow's paradox in the literature [6,7].

Using the concept of the coincidence of lines of velocity and stress discontinuity [8], a new solution is proposed which is admissible for indentation by obtuse-angled wedges. There is no discontinuous jump in load at the transition from 


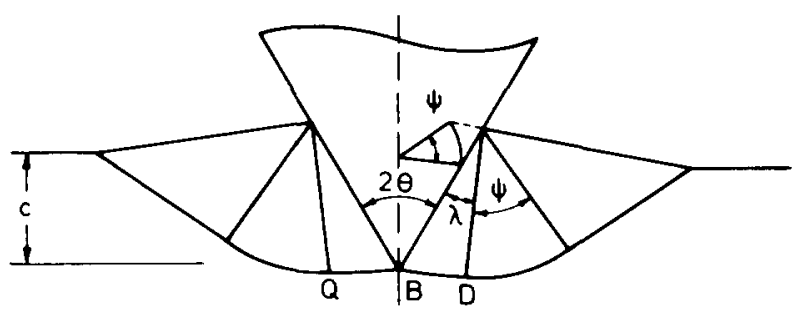

(a)

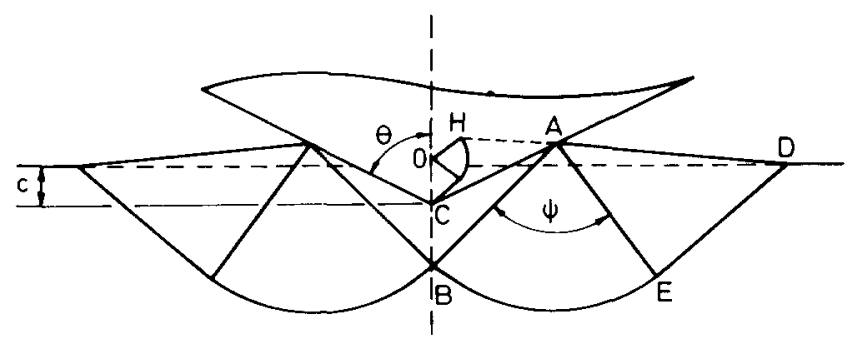

(b)

Fig. 1(a) Slip-line field solution admissible when $\theta-\lambda \leqslant \pi / 4$, (b) Dead metal cap solution used when $\theta-\lambda \geqslant \pi / 4$.

the non-dead metal cap solution to the proposed solution. Therefore, Haddow's paradox does not exist when the new solution is used.

\section{NEW SOLUTION FOR OBTUSE-ANGLED WEDGE INDENTATION}

The slip-line field shown in Fig. 1(a) is statically admissible as long as angle $D B Q$ is not less than $\pi / 2$. In terms of $\theta$ and $\lambda$ this condition is

$$
\theta-\lambda \leqslant \pi / 4
$$

when angle $D B Q$ is less than $\pi / 2$ the rigid material in the wedge bounded by slip-lines $B D$ and $B Q$ is overstressed. In these cases the dead metal cap solution has been used.

The proposed solution is shown in Fig. 2. When angle $D B Q$ is less than $\pi / 2$ the segments $B D$ and $B Q$ are no longer characteristics (weak discontinuities) but become stress discontinuities (strong discontinuities). Also the angle $A D B$ is greater than $\pi / 2$ because $A D$ is still a $\beta$-line and $B D$ is a stress discontinuity. It is clear that when angle $D B Q$ equals $\pi / 2$ both solutions coincide.

\section{ADMISSIBILITY OF PROPOSED SOLUTION}

The condition for the transition from the non-dead metal cap solution to the new solution must be deduced using Hill's generalized overstressing criterion [9] since $B D$ and $B Q$ are stress discontinuities. Referring to Fig. 2, Hill's criterion requires

$$
\cos 2 \zeta-\cos 2 \delta \geqslant 0 \text { when } D \hat{B} Q \leqslant \pi / 2 .
$$

Therefore the minimum angle $\delta$ satisfying Hill's criterion is

$$
\delta=\zeta
$$

Following Hill's analysis, the shear stresses on $B D$ and $B Q$ in the wedge bounded by $B D$ and $B Q$ are $\tau_{1}$ and $\tau_{2}$ where $\tau_{1}=-\tau_{2}$ and

$$
\tau_{1,2}= \pm k \sin 2 \zeta
$$

where $\zeta$ lies between zero and $\pi / 4$

Because the stress state in triangle $A B D$ is uniform and below $B D$ the material is rigid, $B D$ is straight. From Hencky's equations, in triangle $A B D$

$$
\sigma=k[1+2 \psi+\sin 2\{\pi-(\delta+\theta-\lambda)\}]
$$

and

$$
\tau=k \cos 2(\delta+\theta-\lambda) \text {. }
$$




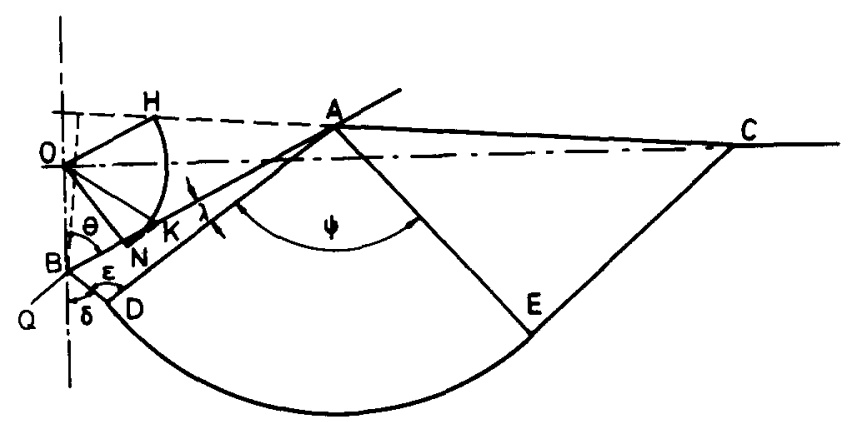

FIG. 2. Proposed solution with lines of stress discontinuity $B D$ and $B Q$ admissible when $\theta-\lambda \geqslant \pi / 4$ except when $\lambda=0$.

For a stress discontinuity the following condition must be satisfied,

$$
\left[\sigma_{i j} n_{j}\right]=0
$$

where $\sigma_{i j}$ is the stress tensor and $n_{i}$ is the normal to the discontinuity surface. Comparing equations (4) and (5) and introducing (3), it is found that angle $\delta$ is given by,

$$
\delta=\frac{3 \pi}{8}-\frac{(\theta-\lambda)}{2}
$$

For the non-dead metal cap case shown in Fig. 1(a) the tip semi-angle $\delta_{w}$ is given by,

$$
\delta_{w}=\frac{\pi}{2}-(\theta-\lambda)
$$

Fig. 3 shows tip semi-angle vs $(\theta-\lambda)$. At the point of transition $\delta$ and $\delta_{w}$ coincide and for larger values of $(\theta-\lambda), \delta>\delta_{w}$

For point $C$ in Fig. 2 to be at the same height as the original undeformed surface requires

$$
h \cos \theta-c=\frac{\sqrt{ } 2 h \sin (\theta+\delta) \sin (\theta-\lambda-\psi+\pi / 4)}{\sin (\theta+\delta-\lambda)} .
$$

If equation (9) is substituted into equation (10), then this is coincident with the equation for the solution shown in Fig. 1(a).

A further geometrical condition may be derived from the requirement for geometrical similarity. This similarity condition is derived with the help of the unit diagram shown in Fig. 2.

The foci are a circular arc $H N$ and point $K . O H$ is parallel to $E C$ and $O N$ is parallel to the tangent to the arc $D E$ at $D$. Also $O K$ is parallel to $B D$ because only a tangential velocity discontinuity is permissible. Points $H, K$ and arc $H N$ are the foci for elements in $A E C, A B D$ and fan $A D E$, respectively. Geometrical similarity requires that points $K$ and $H$ lie on $A B$ and the extension of $A C$, respectively. Point $K$ obviously lies on $A B$. For point $H$ to lie on $A C$ extended requires,

$$
h \cos (\lambda+\psi-\pi / 4)=\frac{c \sin \theta \sin \epsilon}{\sqrt{2} \sin (\lambda+\epsilon)}+c \cos (\theta-\lambda-\psi+\pi / 4)
$$

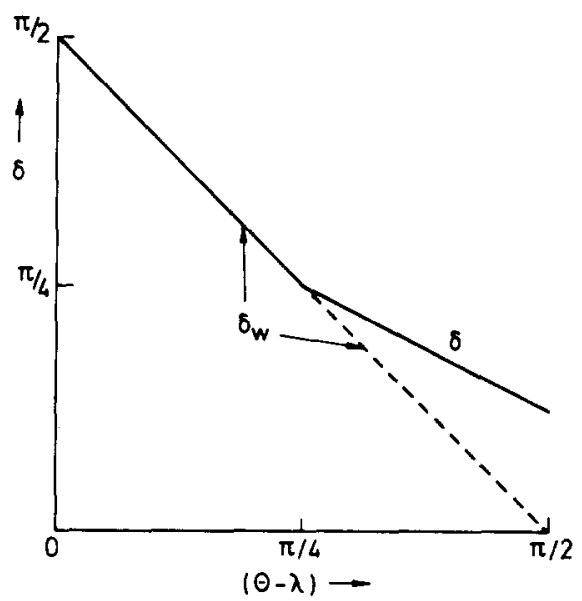

FIG. 3. Relationships between the tip semi-angle $\delta$ and $(\theta-\lambda)$ for the non-dead metal solution $\left(\delta_{w}\right)$ and the proposed solution $(\delta)$. 

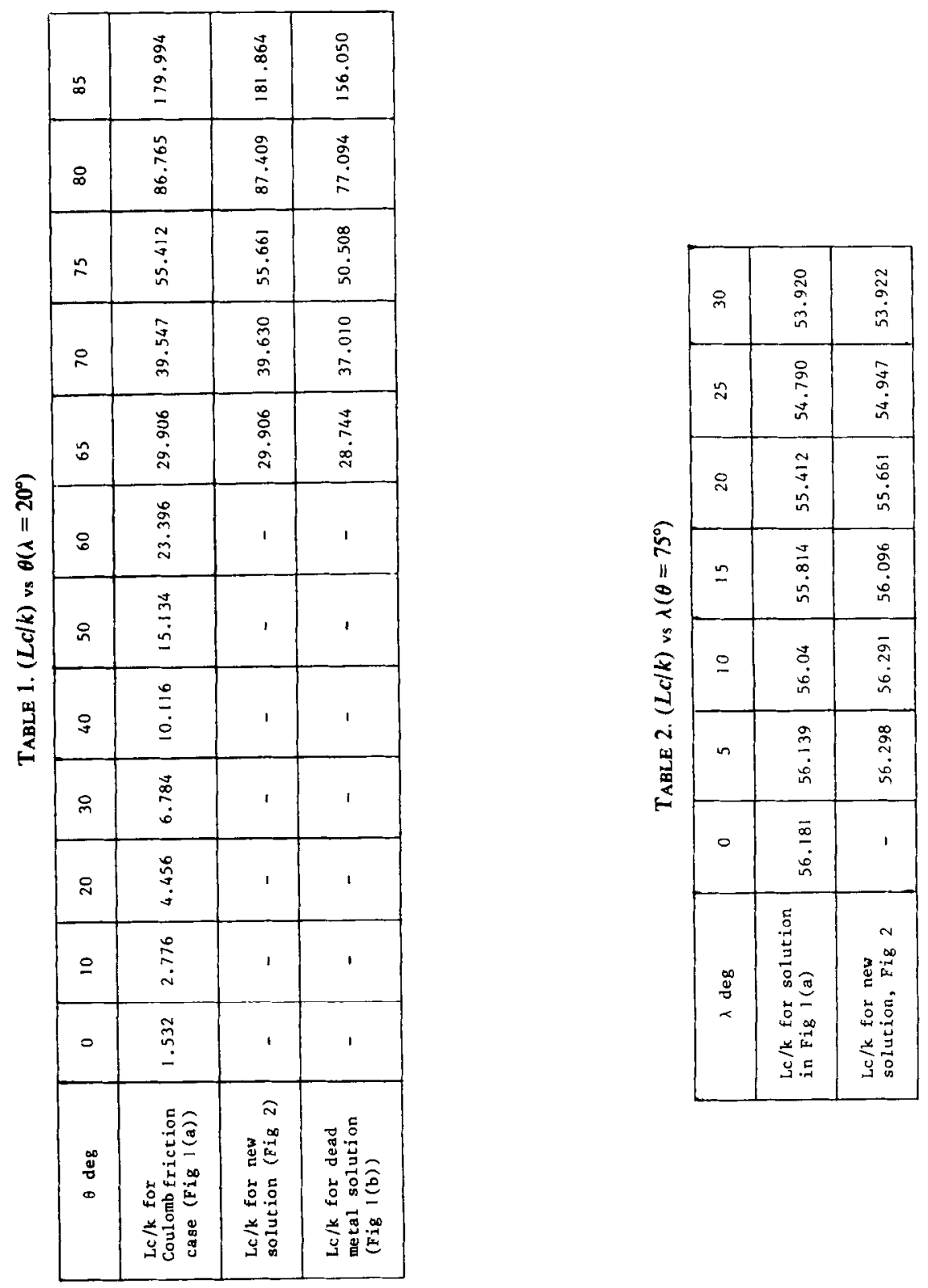
where $\epsilon=\theta+\delta-\lambda$. If $\epsilon=\pi / 2$ is substituted into this equation, then it coincides with the condition for the Coulomb friction solution shown in Fig. 1(a).

The final check for this new solution is that the plastic work rate across the stress discontinuities is not negative. It is clear that deforming material below $B D$ undergoes positive plastic work on crossing $B D$. $A D$ is only a velocity discontinuity and is a characteristic. Green[10] has discussed these discontinuities and concluded "a velocity discontinuity is only possible (for a positive plastic work rate), if the $\alpha$ - (and $\beta$-) direction at 0 (on the discontinuity) coincide, the slip-lines are continuous and there is no stress discontinuity." Obviously, $A D$ satisfies all these conditions.

The solution described here can be used for any wedge within and beyond the critical condition $\theta-\lambda=\pi / 4$ except in cases where $\lambda=0$. In this latter case $D$ coincides with $B$ and this would change the properties of the characteristic $D E$.

It may be shown that the load per unit length of the wedge is given by,

$$
L=\frac{2 h k}{c}\{(1+2 \psi) \sin \theta+\cos (\theta-2 \lambda)\} .
$$

\section{COMPARISON OF LOADS FOR THE THREE SOLUTIONS}

Table 1 shows the load variations with increasing wedge semi-angle $\theta$ at a fixed frictional condition $\left(\lambda=20^{\circ}\right)$ for the three types of solution. The first line are the loads required for the Coulomb friction solution. Although this solution is physically meaningless beyond $\theta=65^{\circ}$ because of violation of Hill's overstressing criterion, the data are quoted for comparison. The second line in Table 1 shows the loads for the proposed solution. It is clear that the transition $\theta-\lambda=\pi / 4$, here corresponding to $\theta=65^{\circ}$, the loads for both solutions coincide. Also for comparison the load values for the dead metal case are given in the table.

Table 2 shows the variation in the loads for the Coulomb friction case and the new solution with $\lambda$ for a fixed wedge semi-angle, $\theta=75^{\circ}$.

It is worth noting here the comments of Johnson et al.[4] concerning the Coulomb friction and the dead metal solutions: "since the deformed surface is not the same in the two solutions, it is not possible to state that the lower load is a better upper bound." The reason for this is that the uniqueness theorems cannot be applied because the form of part of the boundary is part of the solutions. The same observation is true when comparing the dead metal cap solution with the new solution.

\section{CONCLUSIONS}

A slip-line field solution has been proposed for obtuse-angled wedge indentation. This solution includes lines of stress discontinuity and has been proposed to replace the often used dead metal cap solution. The new solution has been shown to be statically and kinematically admissible for case in which $\theta-\lambda>\pi / 4$. Using the new solution it has been shown that Haddow's paradox does not exist.

Acknowledgements-The authors wish to thank Prof. W. Johnson, University Engineering Department, Cambridge for helpful discussions.

\section{REFERENCES}

1. J. Grunzweig, I. M. Longman and N. J. Petch, J. Mech. Phys. Solids 2, 81 (1954).

2. R. HILl, E. H. LeE and S. J. Tupper, Proc. Roy. Soc. A188, 273 (1947).

3. R. HILl, The Mathematical Theory of Plasticity, p. 220 Clarendon Press, Oxford (1950).

4. W. Johnson, F. U. Mahtab and J. B. Haddow, Int. J. Mech. Sci. 6, 329 (1964).

5. J. B. HADDow, Int. J. Mech. Sci. 9159 (1967).

6. A. SHINDO, Memoirs Faculty of Engineering. Kobe University No. 18, 65 (1972).

7. B. DodD and K. OsaKada, Int. J. Mech Sci. 16, 931 (1974).

8. Y. L. BAI, W. JOHNSON and B. DODD, On tangential velocity discontinuities being coincident with stress discontinuities Int. J. Mech. Sci. (In press).

9. R. Hill, J. Mech. Phys. Solids 2, 278 (1954).

10. A. P. Green, Quart. J. Mech. Appl. Math. 6, 223 (1953). 